\title{
Clover fermions in Numerical Stochastic Perturbation Theory
}

\author{
M. Brambilla* \\ INFN Milano-Bicocca and Università di Parma \\ E-mail: michele.brambilladfis.unipr.it \\ Francesco Di Renzo \\ Università di Parma and INFN \\ E-mail: Erancesco.direnzo@fis.unipr.it \\ Marco Guagnelli \\ INFN Pavia and Università di Pavia \\ E-mail: marco.guagnelliepv.infn.it
}

\section{Barbara De Palma}

Università di Pavia

E-mail: barbara.depalma01@ateneopv.it

We present an implementation of clover fermions in Numerical Stochastic Perturbation Theory. As a proof of concept we show two loop computation of critical mass. We stress that any two loop computation only relies on the known value of $c_{S W}$ to one loop. A two loop determination of $c_{S W}$ would be of great interest: we report on the status of our computation of the latter.

The 33rd International Symposium on Lattice Field Theory

14 - 18 July 2015

Kobe International Conference Center, Kobe, Japan

\footnotetext{
* Speaker.
} 


\section{Goal of the project}

In 1993 Di Renzo et al. [1] introduced Numerical Stochastic Perturbation Theory (NSPT) as an alternative to diagrammatic Lattice Perturbation Theory (LPT). Since then its range of applicability increased; in particular at the Lattice 2013 conference the Schrödinger functional formalism [2] and the Wilson flow [3] has been introduced. The current project is the implementation of the clover fermion regularization in NSPT.

As it is well known, in presence of Wilson fermions the lattice QCD action suffers from $O(a)$ artifacts that can be removed provided one add the so called "clover term" in the action:

$$
\mathscr{O}=a^{5} \sum_{x} c_{S W} \bar{\psi}(x) \frac{i}{4} \sigma_{\mu \nu} \hat{F}_{\mu v}(x) \psi(x)
$$

In the former equation $\hat{F}_{\mu \nu}=\frac{1}{8}\left\{\hat{Q}_{\mu \nu}(x)-\hat{Q}_{\nu \mu}(x)\right\}$ is a suitable lattice representation of the gluon field tensor, $\hat{Q}_{\mu v}(x)$ the "clover" product of link variables

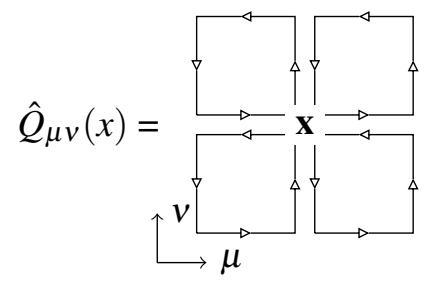

and $c_{S W}=c_{S W}\left(g_{0}\right)$ the Sheikholeslami and Wholert coefficient.

In perturbation theory both the links and $c_{S W}\left(g_{0}\right)$ have to be expanded in series of the coupling: this gives rise to a growing number of diagrams, usually preventing ordinary diagrammatic to reach high orders. By making use of NSPT we are able to overcome this limitation.

In the following we will briefly describe NSPT, then discuss two approaches to the determination of $c_{S W}$ to high orders and finally present a preliminary measurement of the quark critical mass .

\section{The NSPT approach}

A detailed description of the NSPT formulation can be find in 㺻. In brief, the main idea is to add a new degree of freedom, known as stochastic time, to the gauge field and let the system evolve evolve according the Langevin dynamic:

$$
\begin{aligned}
& U(x) \rightarrow U(x, t) \\
& \partial_{t} U(x, t)=\{-i \nabla S[U(x, t)]-i \eta(x, t)\} U(x, t),
\end{aligned}
$$

where $\eta(x, t)$ is a noise satisfying:

$$
\langle\eta(x, t)\rangle=0 \quad\left\langle\eta(x, t) \eta\left(x^{\prime}, t^{\prime}\right)\right\rangle=2 \delta\left(x-x^{\prime}\right) \delta\left(t-t^{\prime}\right)
$$

The gauge fields are then expanded in a power series, to be eventually truncated at the desired order. By trading the differential equations for integral ones one would obtain a diagrammatic approach; in NSPT one uses the lattice regularization of the theory and performs the integration numerically 
on a computer.

As usual fermionic contributions can be included by adding the term

$$
\mathrm{S}_{\mathrm{eff}}=-\operatorname{Tr} \ln M[U]
$$

to the action. This term produces a contribution

$$
\nabla \mathrm{S}_{\mathrm{eff}}=-\operatorname{Tr}\left[(\nabla M[U]) M[U]^{-1}\right]
$$

in the Langevin equation. In order to evaluate this contribution, we observe that

- the trace can be stochastically evaluated inserting a gaussian source $\xi$ s.t. $\left\langle\xi_{i} \xi_{j}\right\rangle_{\xi}=\delta_{i j}$

$$
\operatorname{Tr}\left[(\nabla M) M^{-1}\right]=\operatorname{Re}\left\langle\xi^{\dagger}(\nabla M) M^{-1} \xi\right\rangle_{\xi}
$$

- the Lie derivative $\nabla M$ can be analytically computed.

We are then left with the computation of $M^{-1}$. Since the perturbative expansion of $U$ induces an expansion of the Dirac operator

$$
M[U]=\sum_{n=0} \beta^{-n / 2} M[U]^{(n)}
$$

the inversion $\psi=M[U]^{-1} \xi$ turns into the inversion of a series, that can be iteratively constructed:

- compute $\psi^{(0)}=M^{(0)^{-1}} \xi$

- order by order construct $\psi^{(n)}=M^{(0)}-1\left[\sum_{m=0}^{n-1} M^{(n-m)} \psi^{(m)}\right]$.

According to this scheme the only inversion required is $M^{(0)^{-1}}$, but this can usually be computed analytically. As an example in the case of Wilson fermions the inversion is trivial when performed in momentum space.

In this sense clover fermions are a lucky case: the clover term vanishes at tree-level, and the inversion is the same that one would perform with unimproved Wilson fermions.

\section{Status of the computation}

The final goal of the project is the computation of $c_{S W}$ to higher order. To this end we are considering two approaches: via either the improvement of the quark-gluon vertex or the PCAC mass. We will now briefly discuss the two approaches.

\section{1 quark-gluon vertex improvement}

The on-shell quark-gluon vertex suffers from order $a$ effects, that can be removed by introducing $c_{S W}$. At tree-level the massless quark-gluon vertex reads

$$
\Lambda_{\mu}^{a,(0)}(p, q)=-g T^{a}\left[i \gamma_{\mu}+r\left(\frac{p_{\mu} a+q_{\mu} a}{2}\right)\right]-g \frac{r c_{S W}}{2} T^{a} \sum_{v} \sigma_{\mu v}(p-q)_{v}
$$


Sandwiching $\Lambda_{\mu}^{a}(p, q)_{\alpha \beta}^{i j}$ by the Dirac spinor and making use of the Gordon identity one finds that

$$
\bar{u}(q) \Lambda_{\mu}^{a,(0)}(p, q) u(p)=-g T^{a} \bar{u}(q)\left[i \gamma_{\mu}+\left(1-c_{S W}^{(0)}\right) \frac{a}{2}(p+q)_{\mu}\right] u(p)+O\left(a^{2}\right) .
$$

i.e. setting $c_{S W}^{(0)}=1$ cancels $O(a)$ effects at tree-leevel.

This approach can be used to determine higher orders of $c_{S W}$ by imposing $O(a)$ effects to vanish. In practice the 1-PI vertex function $\Lambda_{\mu}^{a}(p, q)_{\alpha \beta}^{i j}$ can be obtained by Fourier transform and amputation of the quark-gluon vertex function

$$
V_{\mu}^{a}(x, y, z)_{\alpha \beta}^{i j}=\left\langle\psi_{\alpha}^{i}(x) \bar{\psi}_{\beta}^{j}(z) A_{\mu}^{a}(y)\right\rangle,
$$

and the spinors $\bar{u}(q), u(p)$ as solutions of the lattice Dirac equation

$$
\left\{i \gamma_{\mu} \bar{p}_{\mu}+M(\hat{p})\right\} u(p)=0 \quad \bar{u}(p)\left\{i \gamma_{\mu} \bar{p}_{\mu}+M(\hat{p})\right\}=0
$$

Since the higher order equivalent of eq. (3.1) reads

$$
\bar{u}(q) \Lambda_{\mu}^{a,(n)}(p, q) u(p)=-g^{2 n+1} T^{a} \bar{u}(q)\left[\gamma_{\mu} F_{1}+a(p+q)_{\mu} G_{1}+a(p-q)_{\mu} H_{1}\right] u(p)
$$

by measuring (3.1) at different values of $c_{S W}$ we expect to be able to determine order by order the values of $c_{S W}$ that cancel $O(a)$ artifact.

\subsection{PCAC mass improvement}

The PCAC relation $\partial_{\mu} A^{a}=2 m P^{a}\left(A_{\mu}^{a}\right.$ being the isovector axial current, $m$ the quark mass and $P^{a}$ the pseudoscalar current) on the lattice suffers from $O(a)$ effects:

$$
\left\langle\frac{1}{2} A_{\mu}^{a}(x) \mathscr{O}\right\rangle=2 m\left\langle P^{a}(x) \mathscr{O}^{a}\right\rangle+O(a) .
$$

If the theory is on-shell improved, though, $O(a)$ effects are canceled and eq. (3.2) is exact up to $O\left(a^{2}\right)$ effects. More to the point it has been shown [5] that eq. (3.2) can be used as a probe to properly tune $c_{S W}$.

In order to fully improve (3.2) the axial current has to be improved as well: a second coefficient, namely $c_{A}$, has to be introduced. The strategy suggested in [5] requires to measure

$$
\begin{aligned}
& f_{A}=\frac{1}{3} \sum_{x}\left\langle A_{0}^{a}(x) \bar{\psi}(\mathbf{y}) \gamma_{5} \frac{1}{2} \tau^{a} \psi(\mathbf{z})\right\rangle \\
& f_{P}=\frac{1}{3} \sum_{x}\left\langle P^{a}(x) \bar{\psi}(\mathbf{y}) \gamma_{5} \frac{1}{2} \tau^{a} \psi(\mathbf{z})\right\rangle
\end{aligned}
$$

and define a fully improved PCAC mass via

$$
m_{\mathrm{PCAC}}=\frac{\frac{1}{2}\left[\frac{1}{2}\left(\partial_{0}^{L}+\partial_{0}^{R}\right) f_{A}+c_{A} \partial_{0}^{L} \partial_{0}^{R} f_{P}\right]}{f_{p}} .
$$

in the framework of the Schrödinger functional. By measuring $m_{\mathrm{PCAC}}$ with different choices of background fields it is possible to single out $c_{S W}$ and $c_{A}$ contributions and determine the two coefficients. 


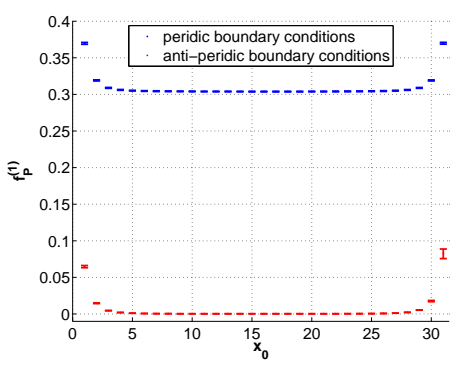

(a)

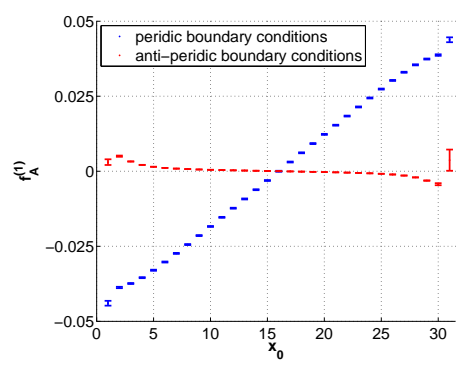

(b)

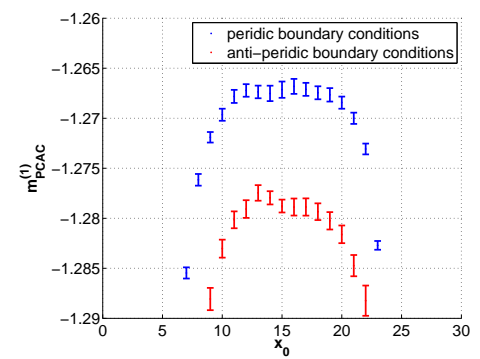

(c)

Figure 1: $f_{P}^{(1)}, f_{A}^{(1)}$ and $m_{\mathrm{PCAC}}^{(1)}$ at finite bare quark mass with periodic (blue) and antiperiodic (red) boundary conditions.

Since fermions are not yet available int NSPT for the Schrödinger functional, we explored the viability of this approach inspecting the signals of $f_{A}, f_{P}$ and $m_{\mathrm{PCAC}}$ on a periodic (and antiperiodic) lattice. Fig. (1) shows $f_{P}^{(1)}, f_{A}^{(1)}$ and $m_{\mathrm{PCAC}}^{(1)}$ averaged over 42 configurations $\mathrm{L}=32$ at fixed stochastic time. Since data still require an extrapolation towards continuum stochastic time we expect that final errorbars grow, nevertheless fig. (10) suggests that we will be able to distinguish between different boundary conditions.

\section{A proof of concept}

In order to show the correctness of the implementation of clover fermions we computed the quark critical mass to up to two-loop for quenched Iwasaki gauge action. The highest order we can compute is currently limited by the order $c_{S W}$ is known up to. A broad description of the computational strategy is described in [6]. We performed the measurement on Iwasaki quenched configurations at different lattice volumes and extrapolated towards the infinite volume limit.

The quark critical mass can be computed as the zero-momentum limit of the diagonal part of the propagator:

$$
\begin{aligned}
\hat{S}\left(\hat{p}, \hat{m}_{c r}, \beta^{-1}\right)^{-1} & =i \hat{p}+\hat{m}_{W}(\hat{p})-\hat{\Sigma}\left(\hat{p}, \hat{m}_{c r}, \beta^{-1}\right) \\
\hat{\Sigma}\left(0, \hat{m}_{c r}, \beta^{-1}\right) & =\hat{m}_{c r}
\end{aligned}
$$

Eq. (4.1, 4.2) have to be intended as expansions in powers of the bare coupling. Since one-loop quark critical mass is known, we plugged it as a counterterm: the one-loop extrapolated quark critical mass is then expected to vanish. Fig. (2a) shows the values of $\hat{m}_{c r}^{(1)}$ at different values of the square momenta $(p a)^{2}$ and different lattice size: $\mathrm{L}=12$ (black), 16 (cyan), 20 (magenta), 24 (red), 32 (blue). In fig. (2b) we plot the zero-momentum extrapolation of $\hat{m}_{c r}^{(1)}$ as a function of the inverse lattice size $L$. Note that the red line in the figure only serves as a guide for the eye. At one-loop we obtained

$$
m_{c r}^{(1)}=-0.001 \pm 0.003
$$

in good agreement with analytical results (remember that since we plugged the counterterm we expect the one-loop critical mass to vanish). 


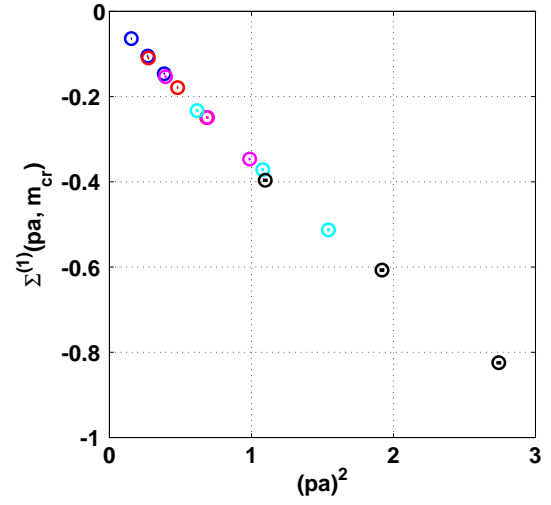

(a)

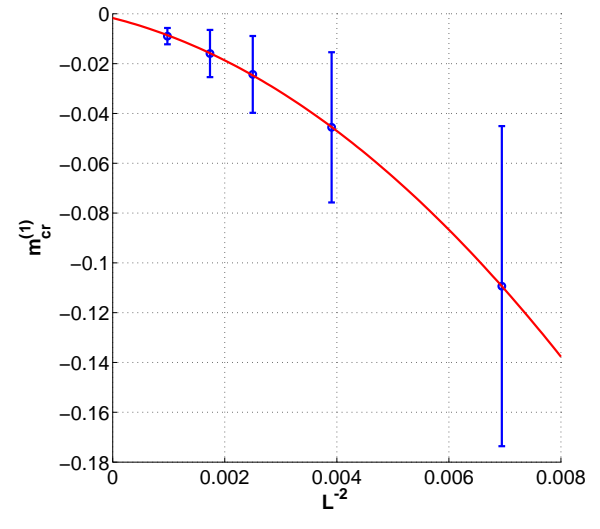

(b)

Figure 2: $\hat{\Sigma}^{(1)}($ pa) measured at different lattice sizes (a): L=12 (black), 16(cyan), 20(magenta), $24($ red) and 32(blue) and its overall approach to the infinite volume limit (b). Red line only serves as a guide for the eye towards $L=\infty$ limit.

We did the same for the two-loop critical mass $\hat{m}_{c r}^{(2)}$ as shown in fig. (3a, 3b). Since in this case the counterterm isn't known our computation gives a first (preliminar) result. At two-loop we obtained

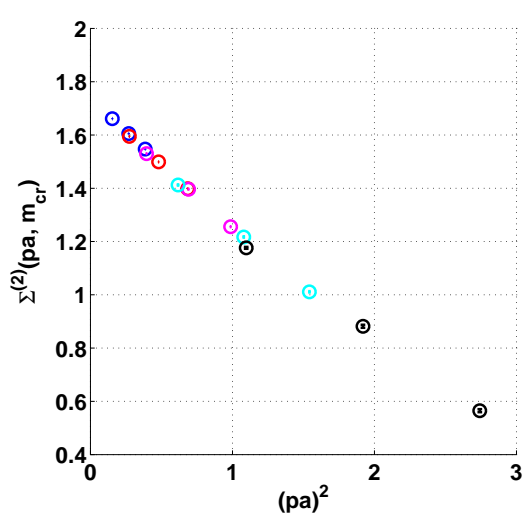

(a)

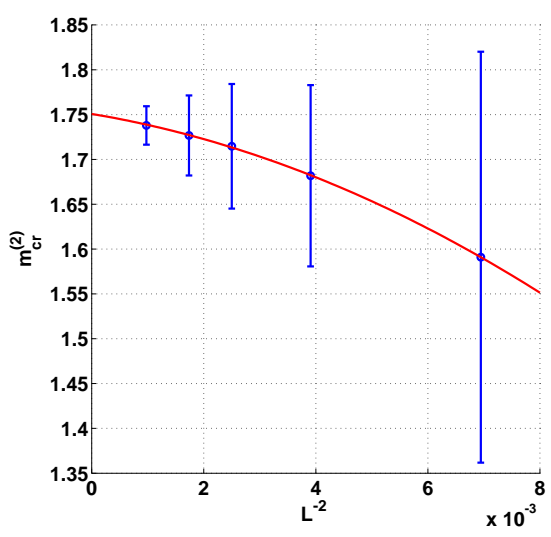

(b)

Figure 3: $\hat{\Sigma}^{(2)}($ pa) measured at different lattice sizes (a): L=12 (black), 16(cyan), 20(magenta), $24($ red $)$ and 32(blue) and its overall approach to the infinite volume limit (b). Red line only serves as a guide for the eye towards $L=\infty$ limit.

$$
m_{c r}^{(2)}=1.75 \pm 0.01
$$

Precision can be further improved considering more momenta and performing a hypercubic lattice expansion [6]. 


\section{Conclusions}

We presented the current status of the implementation of clover fermions in NSPT. In principle any two-loop computations is feasible, since one-loop $c_{S W}$ is known. In order to perform computations at higher order we need to know further terms in the expansion of $c_{S W}$ : such a determination is the main goal of this project. At the moment we are exploring different strategis, trying to understand the most promising.

\section{References}

[1] F. Di Renzo, G. Marchesini, P. Marenzoni and E. Onofri, [hep-lat/9308006].

[2] M. Brambilla, M. Dalla Brida, F. Di Renzo, D. Hesse and S. Sint, PoS Lattice 2013 (2014) 325 [arXiv:1310.8536 [hep-lat]].

[3] M. Dalla Brida and D. Hesse, PoS Lattice 2013 (2014) 326 [arXiv:1311.3936 [hep-lat]].

[4] F. Di Renzo and L. Scorzato, JHEP 0410 (2004) 073 [hep-lat/0410010].

[5] M. Luscher, S. Sint, R. Sommer and P. Weisz, Nucl. Phys. B 478 (1996) 365 [hep-lat/9605038].

[6] M. Brambilla and F. Di Renzo, Eur. Phys. J. C 73 (2013) 12, 2666 [arXiv:1310.4981 [hep-lat]]. 